Viewpoint set

\title{
Ductility and strain hardening in gradient and lamellar structured materials
}

\author{
Xiaolei $\mathrm{Wu}^{\mathrm{a}, \mathrm{b}, *}$, Yuntian $\mathrm{Zhu}^{\mathrm{c}}$, Ke $\mathrm{Lu}^{\mathrm{d}}$ \\ a State Key Laboratory of Nonlinear Mechanics, Institute of Mechanics, CAS, Beijing 100190, China \\ ${ }^{\mathrm{b}}$ School of Engineering Science, University of Chinese Academy of Sciences, Beijing 100190, China \\ ${ }^{c}$ Department of Materials Science and Engineering, North Carolina State University, Raleigh, NC 27695, USA \\ d Shenyang National Laboratory for Materials Science, Institute of Metal Research, Chinese Academy of Sciences, Shenyang 110016, China
}

\section{A R T I C L E I N F O}

\section{Article history:}

Received 2 April 2020

Revised 6 May 2020

Accepted 7 May 2020

Available online 5 June 2020

\section{Keywords:}

Ductility

Strain hardening

Gradient structure

Lamellar structure

Heterogeneous structured materials

\begin{abstract}
A B S T R A C T
Low ductility has long been the bottleneck especially at high strength in metallic structured materials due to conventional forest hardening having little to no effect. Hetero-structuring is an emerging strategy producing superior strength and ductility combination. This Viewpoint article delineates mechanisms for strain hardening and plastic deformation in gradient and lamellar structured materials. Both have typical trans-scale grain hierarchy, leading to sharp mechanical incompatibility and consequent strain gradient at hetero-interfaces during plastic deformation. This induces heterogeneous deformation-induced hardening, along with recovered forest hardening, jointly to improve ductility. The heterogeneous deformationrelated deformation physics sheds lights on the path to designing novel heterostructures particularly for large ductility at high strength.
\end{abstract}

(c) 2020 Acta Materialia Inc. Published by Elsevier Ltd. All rights reserved.
Recently, the heterostructure (HS) of varying kinds attracts growing attention in materials community [1-6]. The HS is usually a specially-designed heterogeneous assembly of certain microstructural components such as grains [7],[8] and twins [9], along with a wide spectrum of mechanical responses. The HS may be roughly divided into two categories in terms of inter- and intra-granular architectural heterogeneities. One is typical of trans-scale grain hierarchy, including gradient structure [2],[7-9]], heterogeneous lamellar structure [10], dual- and multi-phases [11], etc. The other arises from nano-twins [9],[12],[13], short-range order [14], chemical concentration [15],[16], precipitation, etc., all embedded in grain interiors.

The HSs demonstrate an extraordinary strength-ductility combination [1-3]. Of special note is large ductility, almost comparable to that of uniform coarse-grained counterparts [2],[7]. So are HSs even including nanograins (NG) [7] and nanostructures (NS) [8], both with scarcely any ductility. This is indicative of unique effect of heterogeneous deformation due to microstructural heterogeneities [1-6]. These heterogeneities highlight the distinctive role played by strain gradient, which exists at hetero-interfaces in response to plastic incompatibility inside the HSs [3],[4]. Strain gra-

\footnotetext{
* Corresponding author at: State Key Laboratory of Nonlinear Mechanics, Institute of Mechanics, CAS, Beijing 100190, China

E-mail addresses: xlwu@imech.ac.cn (X. Wu), ytzhu@ncsu.edu (Y. Zhu), lu@imr.ac.cn (K. Lu).
}

dient facilitates additional strain hardening especially effective for NG/NS in HSs [4], usually inaccessible to conventional homogeneous microstructures.

Ductility is the perpetual challenge for strength and ductility synergy [1-6]. This is more so especially for HSs with NG/NS of high strength [7],[8]. There is a pressing need, therefore, to understand unique strain hardening for ductility so as to tailor and further design HSs for superior strength-ductility combination. This is the purpose of this View-point paper. Two typical HSs, i.e. gradient structure and lamellar structure, are illustrated in detail to reveal synergistic contribution of both heterogeneous deformationinduced hardening and forest hardening to ductility as well as strength.

\section{Gradient structure}

The gradient structure (GS) refers, in a broad sense, to a continuous change of certain microstructural components in three dimensions of bulk specimen [1],[2]. The microstructural gradient can be grain size [7],[8], twin density [9], chemical concentration [15],[16], and volume fraction of either nano-precipitates or constituent phase [17], etc.

So far most researches focus on the grain-size GS [2],[79],[12],[13],[16-26]. This GS consists of surface layers of either nanograins (NG) or nanostructure (NS) which encircle a coarsegrained (CG) core, with a grain-size gradient in-between. The GS is 


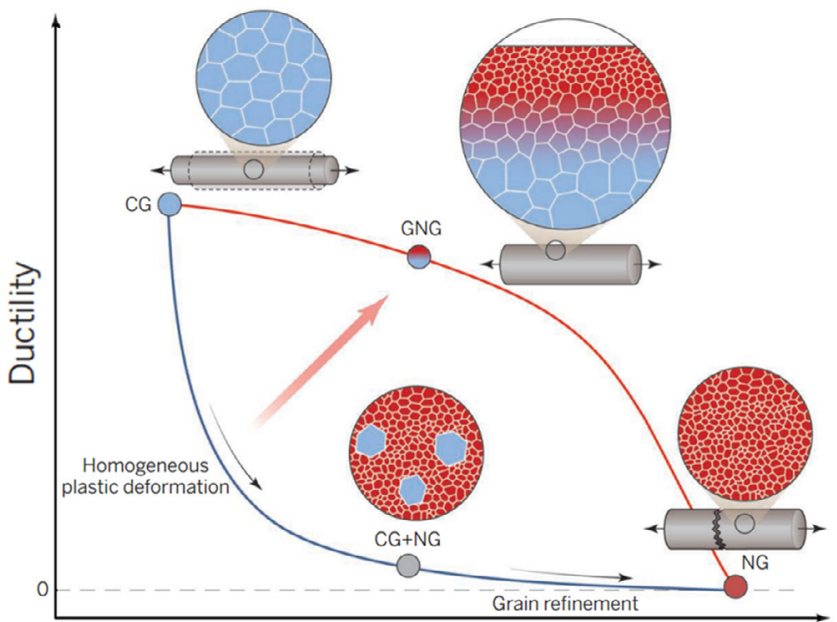

Strength

Fig. 1. Strength-ductility trend in gradient structure [2].

trans-scale, with grain sizes typically spanning 3-4 orders of magnitude from NG to CG [7],[8]. Of special note is polarity of $\left(\sigma_{y}, \varepsilon_{u}\right)$ combination in different layers, where $\sigma_{\mathrm{y}}$ is yield strength and $\varepsilon_{\mathrm{u}}$ is tensile uniform strain. The NS layer shows high $\sigma_{\mathrm{y}}$ but almost absence of $\varepsilon_{\mathrm{u}}$, while the CG core is opposite [7],[8]. This leads to the size effect of plastic deformation, i.e. rapid strain localization in NG/NS layer versus plastic stability in CG layer. Thus, strain incompatibility inevitably appears at the hetero-interfaces upon straining [4],[8], along with vast variation in flow stress according to the classical Hall-Petch relationship. Therefore, the GS leads to typical heterogeneous deformation, which stimulate new deformation physics and consequently, an extraordinary $\left(\sigma_{y}, \varepsilon_{u}\right)$ synergy to alleviate the traditional $\left(\sigma_{\mathrm{y}}, \varepsilon_{\mathrm{u}}\right)$ trade-off, as illustrated in Fig. 1 [2].

The initial focus lies in $\varepsilon_{u}$. The pioneer work by Lu's group [7] first demonstrates an extraordinary $\left(\sigma_{y}, \varepsilon_{u}\right)$ synergy in $\mathrm{GS} \mathrm{Cu}$, see Fig. 2a. This is, actually, the first answer to two long pending asks on whether or not NG is able to act out intrinsic ductility and how big $\varepsilon_{u}$ indeed is. Of special finding is that $\varepsilon_{u}$ of GS $\mathrm{Cu}$ is comparable to that of $\mathrm{CG} \mathrm{Cu}$, while $\sigma_{\mathrm{y}}$ doubles. It turns out that under iso-strain loading, the NG layer in GS bears the same $\varepsilon_{u}$ as that of CG, in sharp contrast to alone NG layer of little $\varepsilon_{u}$. Large $\varepsilon_{u}$ is later reported along with superior $\left(\sigma_{y}, \varepsilon_{\mathrm{u}}\right)$ combinations in interstitialfree (IF) steel (Fig. 2b) [8], Ni [18], and TWIP steel (Fig. 2c) [13]. Recently, a nano-twinned copper was delicately synthesized of both grain size and twin spacing serving as dual gradients [9]. The distinctive benefit is highly increased $\varepsilon_{u}$ over that of only grain-size GS even at much enhanced $\sigma_{\mathrm{y}}$ (Fig. 2d). This indicates the synergistic effect triggered by dual gradients [9].

Several mechanisms for strain hardening were proposed to interpret large $\varepsilon_{u}$ in GS. The first is the mechanically-driven nanograin growth in NG layer upon tensile loading (Fig. 3a) [7],[25]. This recovers partly intra-granular dislocation plasticity in NG layer for operative forest hardening [7]. The second is heterogeneous deformation-induced (HDI) strain hardening [3],[4],[8]. Upon applied straining, plastic incompatibility in trans-scale GS exists at hetero-interfaces where strain gradient appears. The geometrically necessary dislocations (GNDs) are thus produced near heterointerfaces to help with compatible deformation. These GNDs, together with interaction with incident dislocations, induces HDI strengthening and HDI hardening [3],[4], as evidenced by the hysteresis loops [8] and HDI stress (previously termed as back stress) [4],[27]. The third is the nanotwin boundary-mediated strain hardening [1],[9]. In the GS of nano-twin density, strong strain harden- ing occurs due to substantial interaction between twin boundary and dislocations, see Fig. 3c.

With regard to HDI hardening in GS, distinctive dual effects are noteworthy. First, HDI hardening is long-range [4], which plays a key role in accommodating strains at hetero-interfaces. In this sense, HDI hardening is inherent to the trans-scale GS. Second, HDI stress comprises back stress developed in soft CG layer and forward stress in strong NS layer [4]. Specifically, the change of stress state occurs in GS during tensile load [4],[8],[28], which helps with the disentanglement and annihilation of existing dislocations of high density in NS. Namely, forward stress induces the drop at first and rise later in dislocation density in NS, see Fig. 3b. This renders partial recovery of forest hardening in NS during tensile deformation [8],[29], although the NG/NS layer strain softens due to the decrease in total dislocation density [29].

One more mechanism for strain hardening is worthy of mention in GS mainly processed by surface mechanical deformation: the compressive residual stress (CRS)-induced strain hardening [28],[30]. This is usually neglected due to lacking real in situ evidences because the CRS will be released soon after yielding. By using the synchrotron X-ray diffraction in-situ tensile loading in a GS IF steel, the CRS of peak distribution is visible beneath treated surface [28]. The CRS increases at first, reaches the peak and then decreases with depth. Upon yielding, the layer on both sides of CRS peak starts to plastically deform at first while the layer of higher CRS still remains elastic. This leads to the presence of an interface between elastic and plastic zone, near which the GNDs are produced to induce high HDI hardening. With further straining, this interface migrates toward the CRS peak until the layer of CRS peak starts plastic deformation. This leaves behind high density of GNDs as well statistically stored dislocations (SSDs), resulting in both the HDI hardening and forest hardening [8].

Hence, strain hardening in $\mathrm{GS}, \Theta_{\mathrm{GS}}$, is a sum of several contributions, as described in Eq. (1). The first term is an additive effect of individual layer by forest hardening in GS. Note that the NS layer in GS only contribute to $\sigma_{\mathrm{y}}$ but without strain hardening if loaded alone. The second is extra strain hardening by compressive residual stress. The third is the partly recovered forest dislocation hardening due to the change of stress state in NS layer. The last is extra HDI strain hardening by GNDs at hetero-interfaces.

$\Theta_{\mathrm{GS}}=\Sigma \Theta^{\mathrm{i}^{\mathrm{th}}}+\Delta \Theta_{\mathrm{GNDS}}^{C R S}+\Delta \Theta_{\text {forest dislo }}^{\mathrm{NS}}+\Delta \Theta_{\mathrm{GNDs}}^{\mathrm{HDI}}$

The strengthening effect of GS also attracted great interests [8],[9],[31]. The GS features a largely-extended elasto-plastic yielding [32]. Due to the trans-scale grain sizes, $\sigma_{y}$ varies in a wide range based on the classical Hall-Petch relationship. The GS thus successively yields, distinct from yielding in homogeneous microstructures. This leads to an extended elasto-plastic codeformation. The CRS also makes the subsurface layer remain elastic longer than other layers, which further magnifies elasto-plastic incompatibility [28]. The GNDs are produced already at heterointerfaces. Hence, the global $\sigma_{\mathrm{y}}$ in GS is larger than that predicted by the rule-of-mixture [9],[31]. GNDs-induced HDI hardening is responsible for an extra strengthening during elasto-plastic yielding in GS. In other words, GS shows a synergistic strengthening effect, which is also inherent to trans-scale GS.

Last but not least, because of hetero-deformation in GS, a new issue emerges without a very clear picture of whether or not, macroscopically, GS plastically deforms uniformly. It has been long thought that the answer is yes as long as $\varepsilon_{u}$ appears of strain hardening, see the stress-strain curves shown in Figs. 2a and 2b. This hints that the NS layers, along with CG layer, uniformly deforms. However, this is not always true in GS [29]. By using in-situ digitalimaging-correlation (DIC) measurement, it was observed that the NS layer deforms heterogeneously by nucleation and propagation of a macroscopic strain-band (SB) along gage length. Namely, strain 


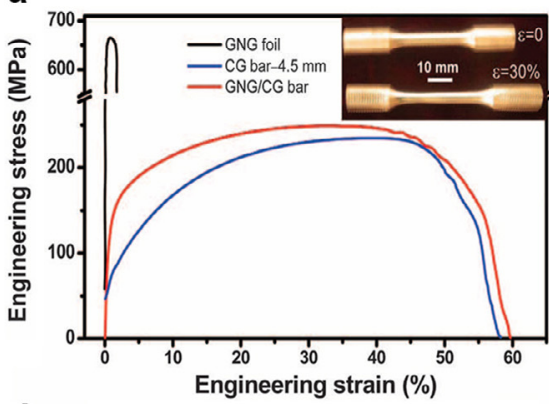

d

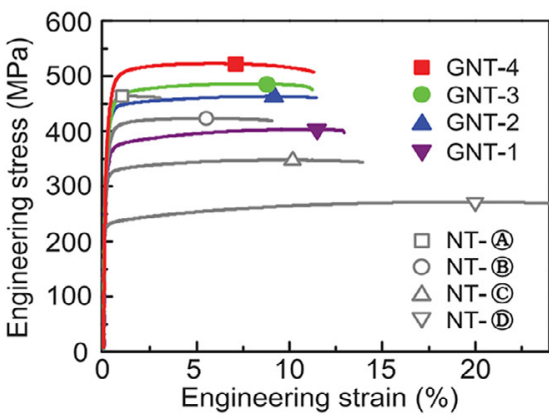

b

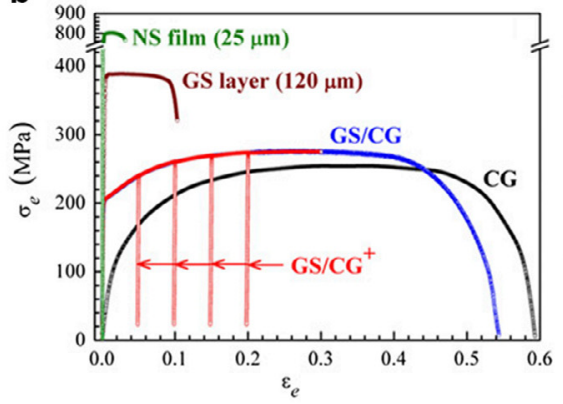

$\mathbf{e}$

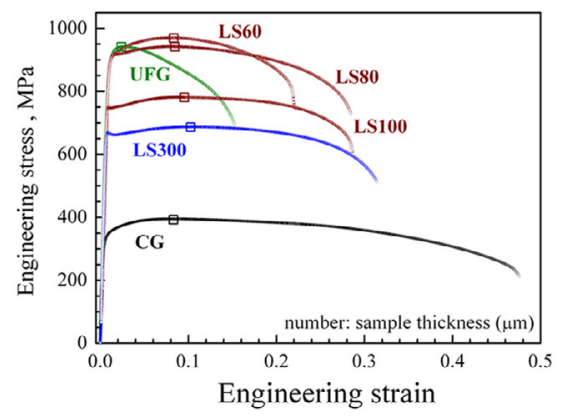

c

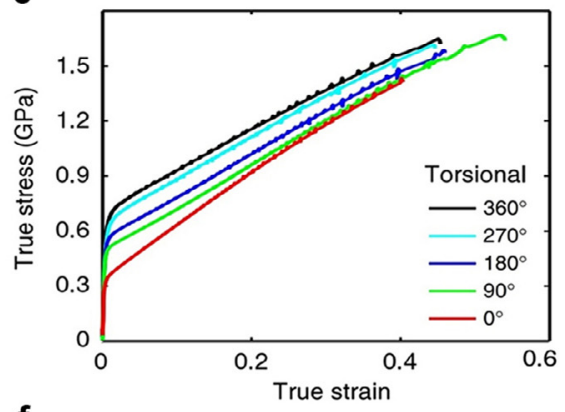

f

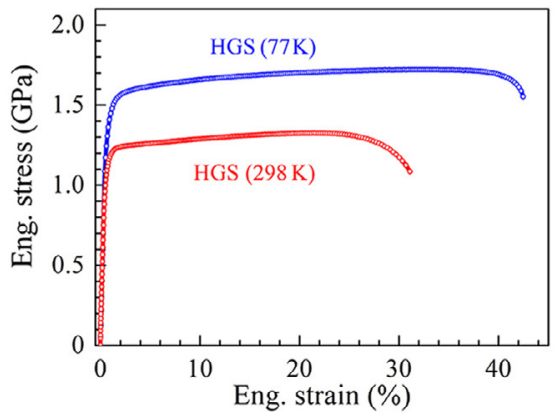

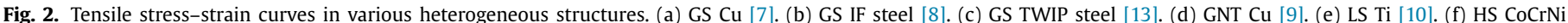

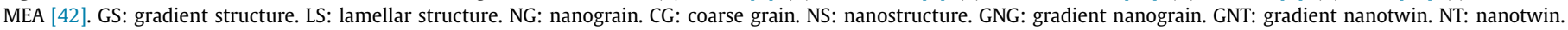
HGS: heterogeneous grain structure.

a

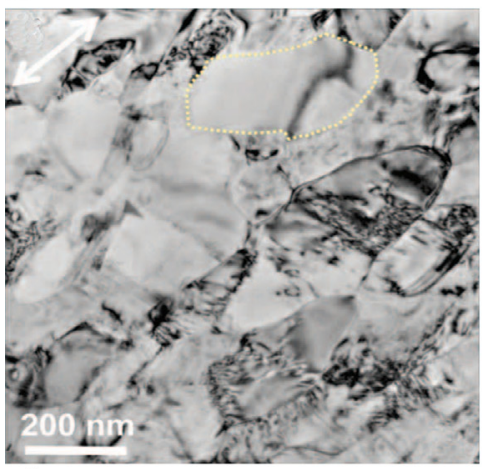

d

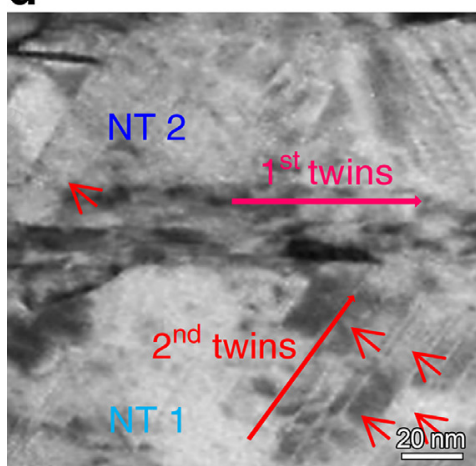

b

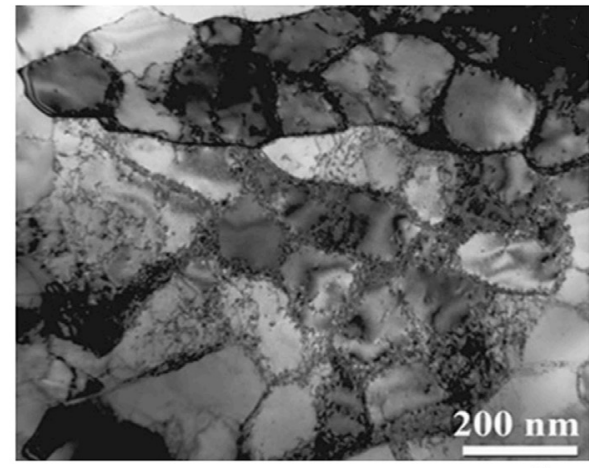

e

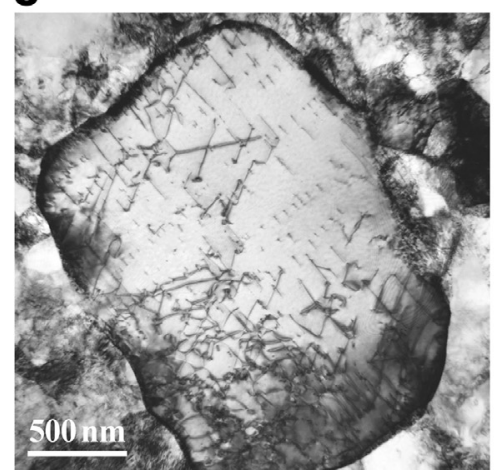

C

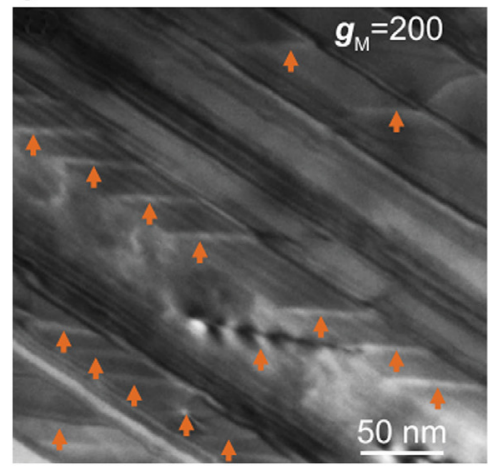

f

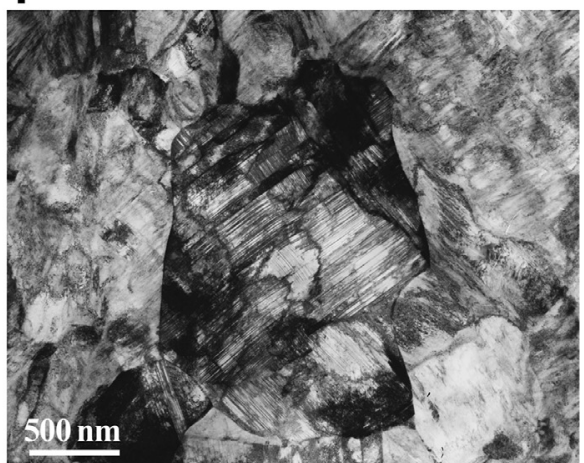

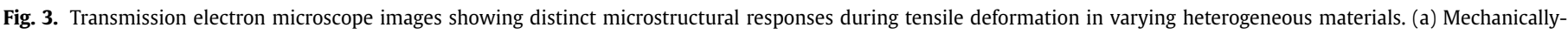

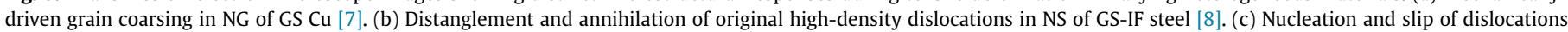

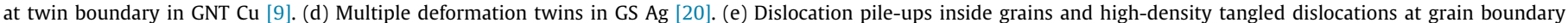
in LS Ti [10]. (f) Dynamic nucleation of nanotwins at grain boundary in LS CoCrNi medium-entropy alloy [42]. 
localization occurs in NS layer, in contrast to uniform deformation in CG core, even both subjected to the same applied strain. Strain gradient of two kinds was detected. One is cross-sectional between NS and CG layer, while the other is axial and in-plane ahead of the propagating SB. Correspondingly, HDI strain hardening is induced. The former prevents SB from propagating into the depth, while the latter slow down and stabilize the propagating SB. Both renders strain delocalization in SB. Further, the micro-hardness (Hv) in SB, as an indicator of strain hardening, drops at first and rises later, yet still with a decrease in overall $\mathrm{Hv}$ eventually. This indicates strain softening in SB, while forest hardening only partly recovered during tensile deformation. Namely, uniform deformation as shown in the stress-strain curve is nothing but pseudo strain hardening in NS layer. It turns out that HDI hardening, along with recovered forest hardening, both synergistically retaining ductility in NS layer. This result helps with understanding heterogeneous deformation in GS from fresh perspectives and to design GS of high mechanical properties. This result also challenges mechanical theoretical analysis and modelling concerning plastic deformation in GS [13],[23],[24]. It should be noted that highly dispersed strain bands were also observed in GS Ni consisting thicker CG layer [33]. This is another way to delocalize strains in the NS layer.

\section{Lamellar structure}

As aforesaid, HDI hardening in HS is ascribed to strain gradient [3],[4], which depends on plastic incompatibility at heterointerfaces among heterogeneities of contrasting plastic responses. Large strain gradient helps with obtaining strong HDI hardening for ductility. The strain gradient-oriented design for hetero-structuring is thus suggested. With regard to the trans-scale HS, the larger discrepancy in grain sizes, the larger strain gradient will be at heterointerfaces due to more drastic plastic incompatibility upon straining. This idea has been early reflected in hetero-structures usually consisting of micro-sized grains embedded in remaining nano-twin bundles and nano-grains by means of dynamic plastic deformation, showing superior $\left(\sigma_{\mathrm{y}}, \varepsilon_{\mathrm{u}}\right)$ combination and strain hardening over those of original CG [34],[35]. However, the role by HDI hardening was not analyzed.

The heterogeneous lamellar structure (HLS) is so far the most effective heterostructure for producing both strengthening and HDI hardening [10]. HLS was reported originally in Ti by partial recrystallization annealing. This HLS Ti consists of soft lamellae of volume fraction of $25 \%$ dispersed in hard NS matrix. Each lamella consists of micron-sized grains (MGs) of $\bar{d}$ of $2.3 \mu \mathrm{m}$. This HLS Ti shows a superior $\left(\sigma_{y}, \varepsilon_{\mathrm{u}}\right)$ combination, see Fig. 3e [10]. Particularly, $\varepsilon_{\mathrm{u}}$ is slightly higher than that of homogeneous CG Ti, while $\sigma_{\mathrm{y}}$ triples, i.e. there is no $\left(\sigma_{\mathrm{y}}, \varepsilon_{\mathrm{u}}\right)$ trade-off.

The strong HDI hardening in HLS Ti is evidenced by the presence of hysteresis loops during unloading-reloading at different tensile strains [10]. Further, back stress hardening, as a component of HDI hardening, is evidenced by GND pile-ups in MG, along with tangled high-density SSDs at hetero-interfaces, see Fig. 2d. HLS Ti is a two-level grain hierarchy consisting of NSs and MGs. The sharp incompatibility renders drastic strain gradient at heterointerfaces for large HDI hardening. Moreover, the HLS with MGs of volume fraction of $25 \%$ shows the same $\sigma_{\mathrm{y}}$ as that in coldrolled NS Ti. This indicates an extraordinary strengthening effect. In addition, the $\left(\sigma_{y}, \varepsilon_{u}\right)$ combination in HLS Ti can be tailored in terms of the size effect. There exists an optimum size for the highest HDI strengthening and work hardening [36-40]. An ideal HLS could produce higher total strain hardening than the CG structure [10]. This is the reason for the simultaneous increase in $\sigma_{\mathrm{y}}$ and $\varepsilon_{\mathrm{u}}$ in HLS Ti. Similar to the GS, HLS Ti features largely extended elasto-plastic transition due to trans-scale grains from MG to NS. As a result, HDI hardening before yielding induces an extra $\sigma_{\mathrm{y}}[10]$.
In addition, the HLS also shows unique advantage over the typical bimodal grain structure [41]. The large grains probably facilitate complete intra-granular dislocation plasticity to operate during tensile deformation [40], with a little or no effect by HDI hardening.

Following the same idea, a three-level grain hierarchy of HS is designed in a nearly equiatomic CoCrNi medium-entropy alloy of much low stacking fault energy (SFE) [42]. This HS, spanning NG $(\bar{d}: \leq 0.25 \mu \mathrm{m})$, ultrafine grains (UFG, $\bar{d}: 0.25-1 \mu \mathrm{m})$ to MG $(\bar{d}: 1-$ $5 \mu \mathrm{m}$ ), provides $\varepsilon_{\mathrm{u}}$ of $20 \%$ and $\sigma_{\mathrm{y}}$ of $\sim 1.2 \mathrm{GPa}$ similar to that of uniform UFG, see Fig. 2 f. Of unique finding is the dynamic generation of nano-twins, which later evolves into NGs at GBs during tensile deformation. This makes it become self-reinforcing three-level HS. Moreover, this three-level HS offers a special advantage over two-level on a smooth strain gradient across neighboring grains through UFG as a transition from NS to MG under the same range of strain gradient. Hence, this HS demonstrates an unprecedented magnitude of back stresses to sustain unusually large, long-range HDI hardening over a wide range of plastic strains [42].

\section{Summary and perspective}

The hetero-structuring strategy is promising for obtaining simultaneous high strength and high ductility so as to upgrade current metallic materials. The relationship between the microstructure and $\left(\sigma_{\mathrm{y}}, \varepsilon_{\mathrm{u}}\right)$ combination suggests deformation physics: transscale grain sizes produce strain gradient at hetero-interfaces, where geometrically necessary dislocations are accumulated to induce HDI strengthening before global yielding and HDI hardening after yielding. The HDI hardening, along with conventional forest hardening, especially recovered in NS, helps with retaining high ductility.

Several fundamental issues need to be further studied for designing heterostructure for superior $\left(\sigma_{\mathrm{y}}, \varepsilon_{\mathrm{u}}\right)$ combination. First, the strain gradient-oriented design of HS is of crucial significance. The novel HS is needed to specialize for ductility at high yield strength in HS containing NS. Second, since HDI strengthening and strain hardening play a critical role in increasing strength and retaining ductility, the HS design of high density hetero-interfaces is thus needed to accumulate GNDs for the best properties. Third, the super-nanograins (s-NG) of usually less than $10 \mathrm{~nm}$ exhibit extremely high strength [43]. HS containing s-NG is hopeful not only to increase yield strength but also to reveal novel deformation physics. Finally, it might be beneficial to couple heterostructures with other strengthening mechanisms for joint strain hardening for better ductility, as demonstrated by the GS coupling with nano-twins [9]. This leads to many new strategies in design HS alloys.

\section{Declaration of Competing Interest}

The authors declare that they have no known competing financial interests or personal relationships that could have appeared to influence the work reported in this paper.

\section{Acknowledgments}

This project was funded by the Ministry of Science and Technology, PR China, 2017YFA02044-01/-02/-03, the NSFC Basic Science Center Program for "Multiscale Problems in Nonlinear Mechanics" (Grant No.11988102), the NSFC (Grant Nos. 11972350), and the CAS (Grant No. XDB22040503).

\section{References}

[1] K. Lu, Nat. Rev. Mater. 1 (2016) 1-13. [2] K. Lu, Science 345 (2014) 1455-1456. 
X. Wu, Y. Zhu and K. Lu/Scripta Materialia 186 (2020) 321-325

325

[3] X.L. Wu, Y.T. Zhu, Mater. Res. Lett. 5 (2017) 527-532.

[4] Y.T. Zhu, X.L. Wu, Mater. Res. Lett. 7 (2019) 393-398.

[5] E. Ma, T. Zhu, Mater. Today 20 (2017) 323-331.

[6] E. Ma, X.L. Wu, Nat. Commun. 10 (2019) 1-10.

[7] T.H. Fang, W.L. Li, N.R. Tao, K. Lu, Science 331 (2011) 1587-1590.

[8] X.L. Wu, P. Jiang, L. Chen, F.P. Yuan, Y.T. Zhu, Proc. Natl. Acad. Sci. U. S. A. 111 (2014) 7197-7201.

[9] Z. Cheng, H. F. Zhou, Q. H. Lu, H. J. Gao, L. Lu, Science 362(2018) 559.

[10] X.L. Wu, M.X. Yang, F.P. Yuan, G.L. Wu, Y.J. Wei, X.X. Huang, Y.T. Zhu, Proc. Natl. Acad. Sci. USA 112 (2015) 14501-14505.

[11] K. Lu, Acta Metall. Sin. 51 (2015) 1-10.

[12] H.N. Kou, J. Lu, Y. Li, Adv. Mater. 26 (2014) 5518-5524.

[13] Y.J. Wei, Y.Q. Li, L.C. Zhu, Y. Liu, X.Q. Lei, G. Wang, Y.X. Wu, Z.L. Mi, J.B. Liu, H.T. Wang, H.J. Gao, Nat. Commun. 5 (2014) 1-8.

[14] Q.J. Li, H. Sheng, E. Ma, Nat. Commun. 10 (2019) 1-11.

[15] R. Roumina, J.D. Embury, O. Bouaziz, H.S. Zurob, Mater. Sci. Eng. A 578 (2013) $140-149$.

[16] H.H. Lee, et al., Scr. Mater. (2020) accepted.

[17] K. Lu, J. Lu, J. Mater. Sci. Technol. 15 (1999) 193-197.

[18] Y. Lin, J. Pan, H.F. Zhou, H.J. Gao, Y. Li, Acta Mater. 153 (2018) 255-264.

[19] W. Chen, Z.S. You, N.R. Tao, Z.H. Jin, L. Lu, Acta Mater. 125 (2017) 413-428.

[20] X.W. Liu, L.G. Sun, L.L. Zhu, J.B. Liu, K. Lu, J. Lu, Acta Mater. 149 (2018) 397-406.

[21] A.Y. Chen, D.F. Li, J.B. Zhang, H.W. Song, J. Lu, Scr. Mater. 59 (2008) 579-582.

[22] J.Y. Kang, J.G. Kim, H.W. Park, H.S. Kim, Sci. Rep. 6 (2016) 26590.

[23] Z. Zeng, X.Y. Li, D.S. Xu, L. Lu, H.J. Gao, T. Zhu, Extreme Mech. Lett. 8 (2016) 213-219.

[24] J. Li, G.J. Weng, S. Chen, X. Wu, Int. J. Plast. 88 (2017) 89-107.

[25] T.H. Fang, N.R. Tao, K. Lu, Scr. Mater. 77 (2014) 17-20.
[26] X.L. Wu, M.X. Yang, F.P. Yuan, L. Chen, Y.T. Zhu, Acta Mater. 112 (2016) 337-346.

[27] M.X. Yang, Y. Pan, F.P. Yuan, Y.T. Zhu, X.L. Wu, Mater. Res. Lett. 4 (2016) $145-151$

[28] M.X. Yang, R.G. Li, P. Jiang, F.P. Yuan, Y.D. Wang, Y.T. Zhu, X.L. Wu, Mater. Res. Lett. 7 (2019) 433-438.

[29] F.P. Yuan, D.S. Yan, J.D. Sun, L.L. Zhou, Y.T. Zhu, X.L. Wu, Mater. Res. Lett. 7 (2019) 12-17.

[30] J.H. Moon, S.M. Baek, S.G. Lee, Y. Seong, A. Amanov, S. Lee, H.S. Kim, Mater. Res. Lett. 7 (2019) 97-102.

[31] X.L. Wu, P. Jiang, L. Chen, J.F. Zhang, F.P. Yuan, Y.T. Zhu, Mater. Res. Lett. 2 (2014) 185-191.

[32] X. L. Wu, unpublished.

[33] Y.F. Wang, C.X. Huang, Y.S. Li, F.J. Guo, Q. He, L.Y. Song, X.L. Wu, R.O. Scattergood, Y.T. Zhu, Int. J. Plast. 124 (2020) 186-198.

[34] F.K. Yan, G.Z. Liu, N.R. Tao, K. Lu, Acta Mater. 60 (2012) 1059-1071.

[35] K. Lu, F.K. Yan, H.T. Wang, N.R. Tao, Scr. Mater. 66 (2012) 878-883.

[36] W.D. Nix, H.J. Gao, J. Mech. Phys. Solids 46 (1998) 411-425.

[37] C.X. Huang, Y.F. Wang, X.L. Ma, S. Yin, H.W. Höppel, M. Göken, X.L. Wu, H.J. Gao, Y.T. Zhu, Mater. Today 21 (2018) 713-719.

[38] R. Yuan, I. Beyerlein, C.Z. Zhou, Mater. Res. Lett. 5 (2017) 251-257.

[39] Y. Xiang, J.J. Vlassak, Acta Mater. 54 (2006) 5449-5460.

[40] Z.M. Li, K.G. Pradeep, Y. Deng, D. Raabe, C.C. Tasan, Nature 534 (2016) 227-230.

[41] Y.M. Wang, M.W. Chen, F.H. Zhou, E. Ma, Nature 419 (2002) 912-915.

[42] M.X. Yang, D.S. Yan, F.P. Yuan, P. Jiang, E. Ma, X.L. Wu, Proc. Natl. Acad. Sci. U. S. A. 115 (2018) 7224-7229.

[43] X. Zhou, X.Y. Li, K. Lu, Science 360 (2018) 526-529. 